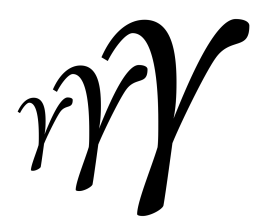

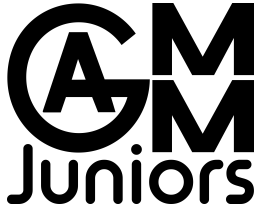

\section{Numerical simulation of fiber-matrix debonding in single fiber pull-out tests}

\author{
Lukas Hoppe $\mathrm{e}^{a, b, \star}$ \\ ${ }^{a}$ Lehrstuhl für Technische Mechanik, Universität Paderborn, Paderborn, Germany \\ ${ }^{b}$ Institute of Composite Structures, German Aerospace Center, Braunschweig, \\ Germany
}

received 04.08.2020, accepted 02.12.2020, published 10.12.2020

\footnotetext{
^ corresponding author: Lukas10Hoppe@web.de

supervisor: Caroline Lüders, Technische Universität Braunschweig, Germany and Christian Willberg, German Aerospace Center, Braunschweig, Germany
}

\begin{abstract}
The present work deals with the numerical crack simulation of fiber-matrix debonding in single fiber pull-out tests. For this purpose, two models are used: a finite element model (FE model) with the cohesive zone approach and a peridynamic model. For calibration a reference experiment is applied. In addition, analytical equations are used for reference values. The influence of the model parameters and the material parameters of the cohesive zone model on the force-displacement curve is investigated. Besides the free fiber length, the critical interface strength, the critical energy release rate as well as the initial interface stiffness have a great influence on the force-displacement curve of the pull-out test. From the crack simulation it can be seen that Mode I has an influence on the crack initiation, but further crack growth after initiation is dominated by Mode II. The FE model can be calibrated in a way that the crack initiation point and the maximum force correspond to the reference experiment. The peridynamic model depicts a comparable crack formation process.
\end{abstract}

Keywords: interface modeling, single fiber pull-out test, fiber reinforced plastics, peridynamic, finite element method, cohesive zone model, glass fiber, epoxy

\section{Introduction}

The interface between the matrix and the single fiber plays an important role not only for the micromechanical behavior of fiber reinforced plastics (FRP) but also for fiber reinforced composites in general, e.g. steel fiber reinforced concrete. Fracture experiments and micromechanical simulations of FRP show that the crack path leads through the interface $[4,13,20,22]$, especially if the composite is loaded perpendicular to the fiber direction. Interface properties are needed for numerical micromechanical simulations. In the present work these interface properties are determined by test results of the single fiber pull-out test. This test is characterized by defined geometric dimensions, a reproducible test sequence and a quasi-static load during the test $[8,30]$. In the pull-out test, a single fiber is embedded in a matrix drop. The fiber is pulled out of the matrix by an applied force. A force-displacement curve is recorded by measuring the applied force and the resulting displacement of the fiber $[8,17,30]$. This test setup is not only used for interface characterization in FRP $[8,17,30]$ but also for other fiber reinforced materials, e.g. steel reinforced cementitious composites [6], poly(vinylalcohol) fibers 


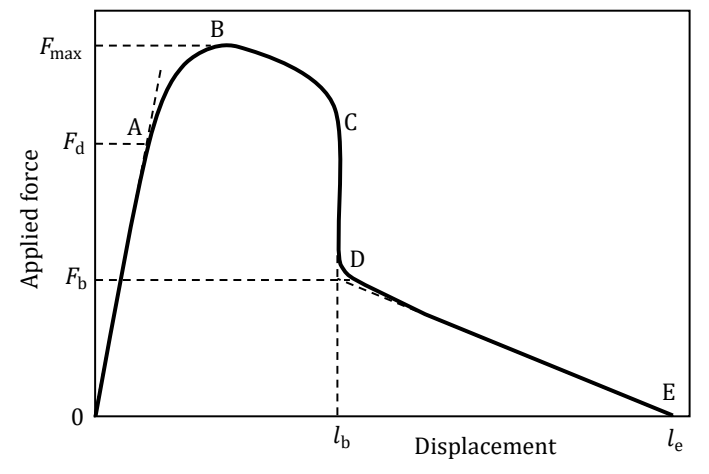

Figure 1 - Schematic force-displacement diagram of a pull-out test [30]

(PVA) reinforced concrete [27, 28] and glass fiber reinforced cement [9].

The local interface properties are to be obtained from the global force-displacement curve of the pull-out test. Figure 1 shows a force-displacement curve, which can basically be divided into five sections with five characteristic Points A to E [30]. In the first section the force rises almost linearly with the displacement up to Point A. This point is considered to be the debonding point, from which a crack in the interface is initiated. This point is characterized by a $C^{1}$ discontinuity in the curve. The force at crack initiation is $F_{\mathrm{d}}$. In the second section, from Point A to B, the progressive zone follows, in which the force increases non-linearly. The stiffness of the progressive phase is composed of the interface stiffness of the remaining fiber-adhesive connection and the frictional force between the already separated interface layers. With increasing crack propagation the separated part grows and the overall stiffness decreases. This results in a decrease of force increase until it peaks at Point $\mathrm{B}$, where the maximum force $F_{\max }$ is reached. From there, the crack growth continues with decreasing force until the fiber is completely debonded in Point $D$. In the third section, from Point $\mathrm{B}$ to $\mathrm{C}$, there is stable crack growth. In the fourth section (from Point $\mathrm{C}$ ) there is unstable crack growth. This is characterized by an abrupt load drop, which stops at Point D with the force of $F_{\mathrm{b}}$ and the displacement of $l_{\mathrm{b}}$. In the fifth section between Point $\mathrm{D}$ and $\mathrm{E}$ there is no more adhesion between matrix and fiber. The force of the fiber is now only transmitted by friction. At Point $\mathrm{E}$ the fiber is completely pulled out of the matrix. The displacement at Point $\mathrm{E}$ is therefore almost equal to the embedded fiber length $l_{\mathrm{e}}$ [30].

For the calibration of the interface properties by pullout test, analytical models are available, which can be used to determine the critical energy release rate or the critical interface strength $[12,15,29]$. The analytical models calculate the force-displacement curve of the pull-out test by fitting the characteristic values. Adjusting the interface parameters, the curve is adapted to the reference experiment [12, 29]. In FE simulations of the single fiber pull-out test, the interface can be modeled by the cohesive zone approach [3, 11]. Within the study of Jia et al. [11] the thermal prestresses, the influence of the free fiber length and the interface friction are not considered. The latter is usually not incorporated within the description of cohesive behavior. That is why Bheemreddy et al. [3] integrate an artificial neural network into the FE model in order to capture the friction dominated last section (D - E) of the force-displacement curve of the pull-out test. A transferability of the analytical parameters to numerical simulations is only partially possible, because other assumptions and especially other interface parameters are used to describe the interface. Therefore, in the present work a calibration is performed directly for two numerical models: An FE model incorporating the cohesive zone approach in ABAQUS $\odot$ and a peridynamic model in Peridigm are used for the simulation of fibermatrix debonding in a single fiber pull-out test. First of all, the influence of the interface parameters on the global force-displacement behavior is investigated. A force-displacement curve [30] is used as reference experiment. The analytical equations from [15, 27, 28, 29, 31] are used to determine reference values for calibration.

The cohesive zone approach, which is used for the implementation of the interface in the FE model, is used in many micromechanical crack simulations of FRP, e.g. [20, 21, 22]. Peridynamics is chosen as an alternative approach. The advantage lies in the formalism, which does not specify a local connection between the elements and allows discontinuities. Thus, more realistic crack processes can be generated than in the methods based on classical local continuum like FEM [18].

\section{Finite element simulation}

\subsection{FE model}

\subsubsection{Material}

In the reference experiment of the pull-out test, the components consist of a glass fiber and, for the matrix, of the epoxy resin "Araldite LY 556" [30]. For the simulation, an isotropic, linear-elastic material model is chosen for both the fiber and the matrix. The material parameters of both constituents at $25^{\circ} \mathrm{C}$ given in [30] are listed in Table 1 . As the cooling of the sample from curing temperature to test temperature is considered in the FE simulations, temperature-dependent material data 


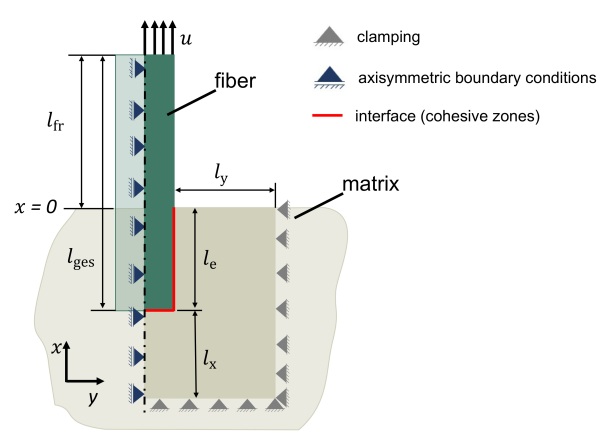

Figure 2-FE model of the pull out test with fiber length $l_{\mathrm{e}}=129 \mu \mathrm{m}$, fiber radius $r_{\mathrm{f}}=6.67 \mu \mathrm{m}$, $l_{\mathrm{x}}=64.5 \mu \mathrm{m}$ and $l_{\mathrm{y}}=64.5 \mu \mathrm{m}$

are used for the matrix. The unknown temperature dependency of the properties of LY556 are approximated by applying the qualitative temperature dependency of another epoxy. The resulting temperature-dependent data for the Young's modulus and the coefficient of thermal expansion (CTE) are given in Table 2. If the given temperature exceeds the tested temperature range, the material properties are hold constant to prevent extrapolation errors.

\subsubsection{Model geometry and boundary conditions}

The FE model depicts the geometry of the reference experiment with the embedded fiber length of $l_{\mathrm{e}}=129 \mu \mathrm{m}$ and the fiber radius of $r_{\mathrm{f}}=6.67 \mu \mathrm{m}$. The modeling approach is based on Marotzke [14]. As the pull-out test sample can be regarded as symmetric and the loading is symmetric, too, axisymmetric modeling is used. Figure 2 shows the setup with boundary conditions, parameter definition, coordinate system and the cohesive zone representing the interface marked in red. A structured mesh with an element size of $1 \mu \mathrm{m}$ and linear axissymmetric elements is selected for meshing. To reduce the computational effort for the simulation, only a matrix section around the fiber is considered. Due to the smaller dimensions of the cutout compared to the real dimensions of the matrix droplet, the geometry of the matrix drop is assumed to be simplified as a cylinder. According to Marotzke [14], a radial $l_{\mathrm{y}}$ and longitudinal distance $l_{\mathrm{x}}$ of the outer cylinder surface from the embedded fiber of half of the embedded fiber length is sufficient to prevent boundary effects from affecting the interface stress state. This results in the matrix droplet dimensions of $l_{\mathrm{x}}=64.5 \mu \mathrm{m}$ and $l_{\mathrm{y}}=64.5 \mu \mathrm{m}$, see Figure 2. The last geometry parameter to be determined is the free fiber length $l_{\mathrm{fr}}$. As in the analytical model of [29], this represents the measured free fiber length in the experiment as well as the compliance of the test setup. It has there-
Table 1 - Material properties of the matrix and the fiber at $25{ }^{\circ} \mathrm{C}[30]$

\begin{tabular}{lcc}
\hline & Epoxy resin & Glass fiber \\
\hline Young's modulus [MPa] & 3200 & 75000 \\
Poisson's ratio & 0.35 & 0.17 \\
CTE $[1 / \mathrm{K}]$ & $57 \times 10^{-6}$ & $5 \times 10^{-6}$ \\
\hline
\end{tabular}

Table 2 - Material properties of the matrix at different temperatures

\begin{tabular}{rcc}
\hline & Young's modulus [MPa] & CTE $[1 / \mathrm{K}]$ \\
\hline $25^{\circ} \mathrm{C}$ & 3200 & $57.0 \times 10^{-6}$ \\
$50^{\circ} \mathrm{C}$ & 2600 & $61.6 \times 10^{-6}$ \\
$100^{\circ} \mathrm{C}$ & 2000 & $65.3 \times 10^{-6}$ \\
$125^{\circ} \mathrm{C}$ & 1500 & $65.3 \times 10^{-6}$ \\
$150^{\circ} \mathrm{C}$ & 500 & $65.3 \times 10^{-6}$ \\
$160^{\circ} \mathrm{C}$ & 200 & $65.3 \times 10^{-6}$ \\
\hline
\end{tabular}

fore to be determined indirectly by the initial slope of the force-displacement curve of the pull-out test.

In Figure 2 the axis of symmetry and the symmetry boundary conditions are defined on the left-hand edge. At the lower and right edge of the matrix a fixed bearing is applied. At the top of the fiber end at $x=l_{\mathrm{fr}}$ a displacement $u$ in positive $x$-direction is defined. Before the displacement is applied, the thermal prestresses due to specimen curing are simulated. It can be assumed that the specimen is in a stress-free state at $128^{\circ} \mathrm{C}$ (curing temperature [30]) and is cooled down to the test temperature of $25^{\circ} \mathrm{C}$. Within this cooling step, the clamping and the axisymmetric boundary conditions are the same as for the pull-out step. The interface between the fiber and the matrix is modeled by the cohesive zone approach.

\subsubsection{Cohesive zone approach}

In this paper the surface-based implementation of the cohesive zone model by ABAQUS $\odot$ is used. The behavior of the cohesive zone is represented by the tractionseparation law [1]. It describes the stiffness and failure behavior of the interface. The bilinear tractionseparation law used is shown in Figure 3.

The contact stress $t_{i}$ is plotted over the contact separation $\delta_{i}$. The initial stiffness $K_{\mathrm{p}, i}$ of the cohesive zone represents the ratio of the contact stress to the separation before damage occurs. A relative displacement of the contact surfaces results in a contact stress in the cohesive zone. If the critical contact stress $t_{i}^{0}$ is reached at the critical displacement $\delta_{i}^{0}$ defined, damage is initiated. 


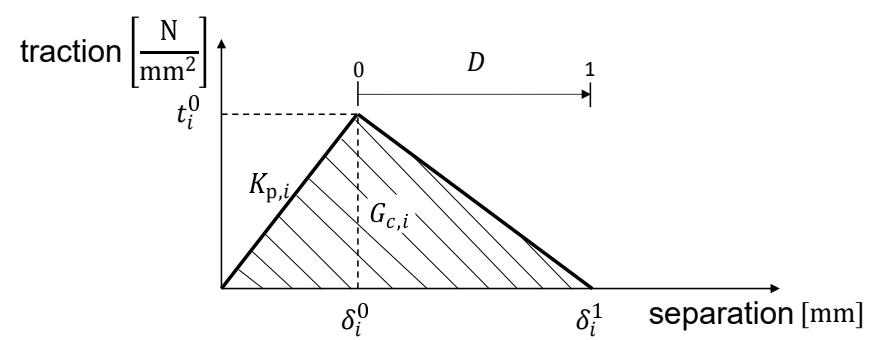

Figure 3 - Bilinear traction-separation law by [1]

Figure 3 represents the traction-separation law behavior for a single fracture mode. If one fracture mode $i$ is acting alone, the crack is initiated at $t_{i}^{0}$. For considering crack initiation under mixed mode conditions, the quadratic stress criterion defined by

$$
\left[\frac{\left\langle t_{I}\right\rangle}{t_{I}^{0}}\right]^{2}+\left[\frac{\left\langle t_{I I}\right\rangle}{t_{I I}^{0}}\right]^{2}+\left[\frac{\left\langle t_{I I I}\right\rangle}{t_{I I I}^{0}}\right]^{2}=1
$$

is used. Based on the contact stress in the different fracture modes and the corresponding critical contact stresses, this criterion calculates an equivalent value and crack is initiated if this value reaches one. Thus, for the case of a mixed mode loading, looking at the tractionseparation law of a single fracture mode, crack initiation could start before the contact stress $t_{i}$ reaching $t_{i}^{0}$. When the crack is initiated based on the quadratic stress criterion, with further increased displacement, the damage grows and the interface stiffness decreases. When the maximum displacement $\delta_{i}^{1}$ is reached, the contact surfaces are completely separated. The damage can be measured by the damage parameter $D$, which is defined by

$$
D=\frac{\delta_{i}^{1}\left(\delta_{i}-\delta_{i}^{0}\right)}{\delta_{i}\left(\delta_{i}^{1}-\delta_{i}^{0}\right)} .
$$

Its value is zero before crack initiation and one when the surfaces are completely separated. Thus, as soon as the $D$ becomes greater than zero, the cracking and degrading process starts, and $D=0$ indicates that not load is transferred anymore. The debonding criterion is defined by the energy release rate $G_{\mathrm{c}, i}$. The critical energy release rate results from the area integral under the curve of the traction-separation law.

For each fracture mode $i=\mathrm{I}$, II, III a traction-separation law is defined. Thus, for each fracture mode the critical energy release rate $G_{\mathrm{c}, i}$, the initial interface stiffness $K_{\mathrm{p}, i}$ and the critical interface strength $t_{i}^{0}$ must be specified.

\subsection{Cracking}

In this section, the crack process during the pull-out test simulated using the FE model is presented. For this cal-

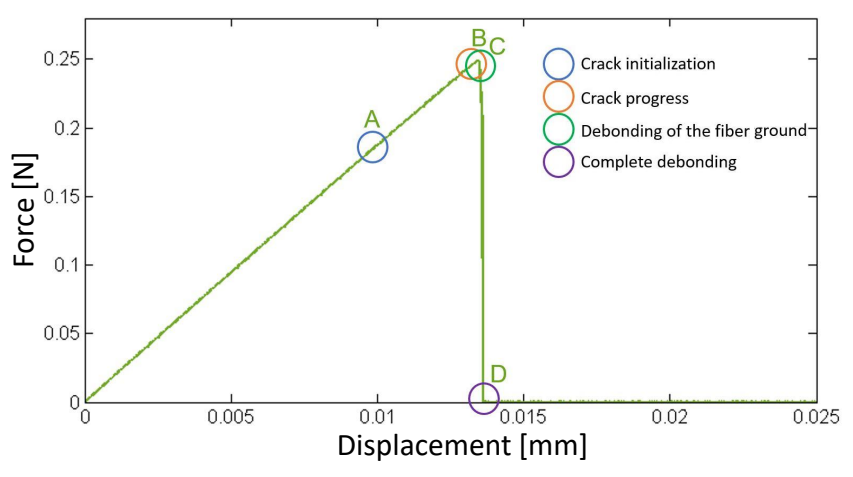

Figure 4 - Force-displacement diagram and characteristic points of crack propagation

culation, the model parameters obtained by calibration Variant B in Section 2.4.2 are used.

For the visualization of the crack propagation the damage parameter $D$ is used, which is called "CSDMG" in ABAQUS $\odot$. This variable is shown in Figure $5-8$ for the different characteristic Points A to D (see Figure 1) of the crack propagation process. These characteristic points are assigned to the global force-displacement curve in Figure 4.

Figure 5 shows the Point A where the fracture starts. From this point, the maximum crack variable in the whole model is greater than zero. Crack initiation takes place at the interface position $x=0$, see Figure 2. At this point, Mode I prevails, caused by the radial stress component [14]. Please note that the deformation in this figure is highly scaled so that separation between fiber and matrix appears larger. Additionally, a small amount of separation until $\delta_{i}^{0}$ is allowed before damage starts, see traction-separation law in Figure 3. That is why the damage variable is still zero for regions that seems to be separated, but they are still bonded.

The crack continues to grow in negative $x$-direction due to the increasing load, see Figure 6. Except from the fiber ground the shear stresses in axial direction dominate in the interface. Therefore, the crack propagation during the pull-out test is mainly Mode II controlled. Mode III does not occur because the corresponding shear stress is negligible due to the axissymmetric setup.

Shortly after the force maximum at Point B, debonding occurs at the fiber bottom, see Figure 7. The crack at the fiber ground is controlled by Mode I. The forcedisplacement curve does not show any significant influence of the debonding of the fiber ground on the curve. This confirms that Mode I has no major influence on the force-displacement curve of the pull-out test.

Figure 8 shows the crack state at Point D. This is the point at which the fiber is completely separated from the matrix. The fracture variable is $D=1$ in the com- 


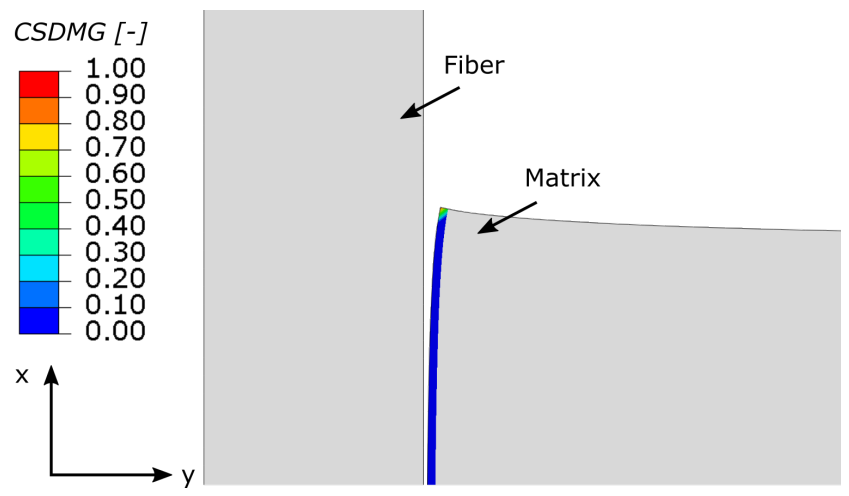

Figure 5 - Cracking status at crack initalisiation (A)

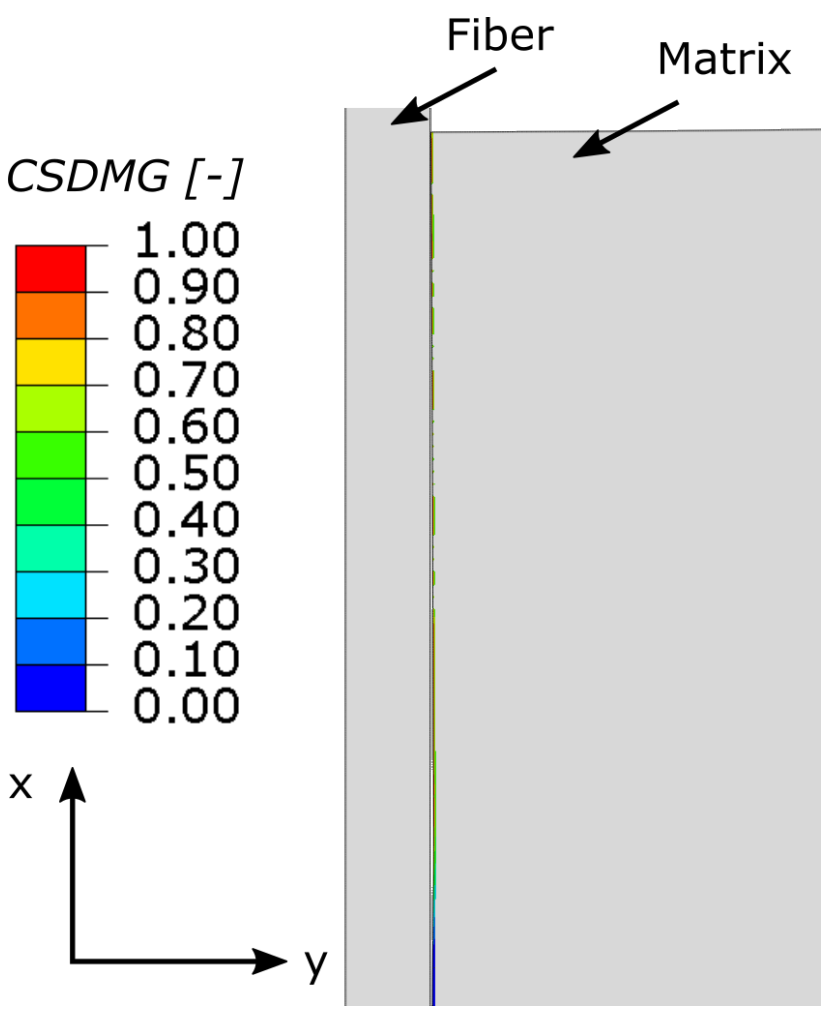

Figure 6 - Cracking status at crack progress (B)

plete interface. Since no frictional forces are modeled in the traction-separation law and no normal force due to surface roughness acts between the separated surfaces, the force drop at Point D reaches $0 \mathrm{~N}$, see Figure 4.

\subsection{Parametric study}

The influence of the traction-separation law parameters on the force-displacement curve of the pull-out test is investigated. For the parameter study, the quadratic stress criterion, a viscous regularization parameter of $10^{-4}$ and the mixed-mode behavior according to Benzeggagh and Kenane [2] with the mixed-mode coefficient $\eta=2.5$ is chosen for the cohesive zone model. The reference value of the provisional free fiber length is $l_{\mathrm{fr}}=365 \mu \mathrm{m}$. The thermal prestresses are neglected in the parameter study.

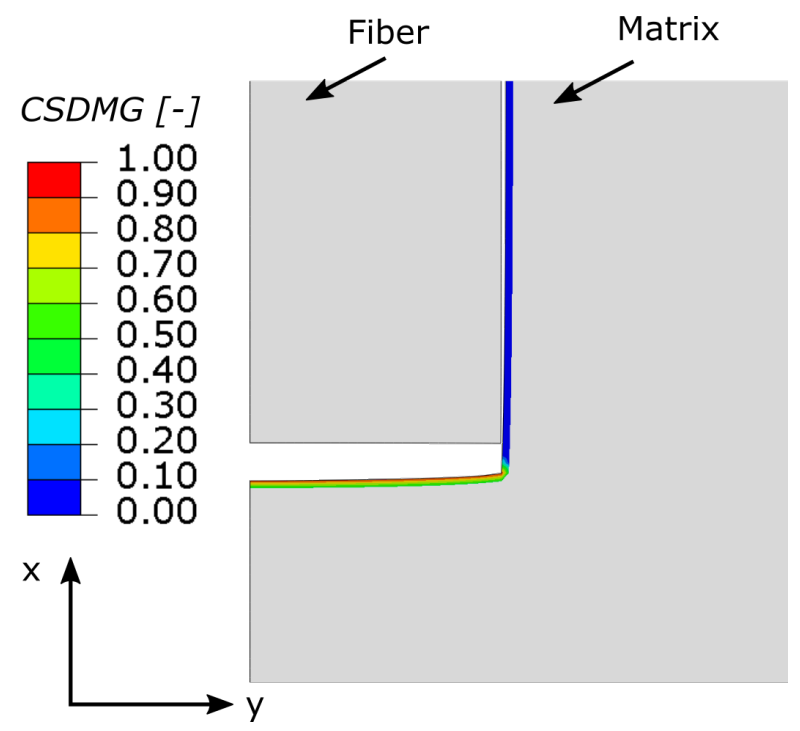

Figure 7 - Cracking status at debonding of fiber ground (C)
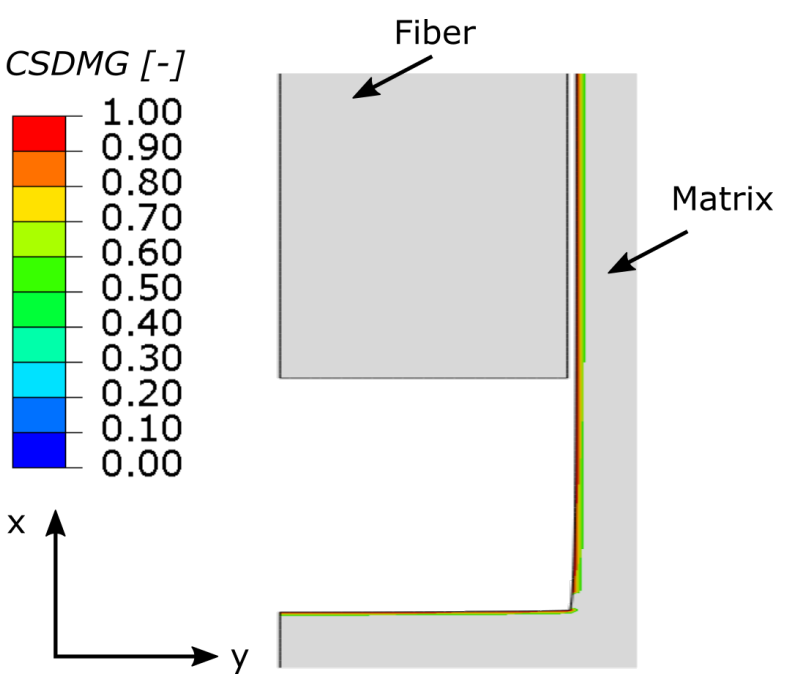

Figure 8 - Cracking status at complete debonding (D)

The parameters to be investigated are the critical energy release rate $G_{\mathrm{c}, i}$, the interface strength $t_{i}^{0}$ as well as the initial interface stiffness $K_{\mathrm{p}, i}$. They are specified equal for each fracture mode in order to reduce the effort of the parameter study. Table 3 shows the parameter variation considered within the study. The bold numbers are the reference values of the parameters.

\subsubsection{Critical energy release rate}

The influence of the critical energy release rate $G_{\mathrm{c}, i}$ on the force-displacement curve is shown in Figure 9. The critical energy release rate has no influence on the initial gradient of the force-displacement curve until crack initiation. According to the traction-separation law, the critical energy release rate only has an influence on the degradation process. That is why for all values of the crit- 
Table 3 - Variation of the traction-separation law parameters with reference values (bold)

\begin{tabular}{llcccc}
\hline \multicolumn{3}{c}{ Parameters } & \multicolumn{4}{c}{ Range of values } \\
\hline$G_{\mathrm{c}, i}$ & {$[\mathrm{~N} / \mathrm{mm}]$} & 0.04 & 0.2 & $\mathbf{0 . 4}$ & 0.8 \\
$K_{\mathrm{p}, i}$ & {$\left[\mathrm{~N} / \mathrm{mm}^{3}\right]$} & $50 \times 10^{3}$ & $\mathbf{1 0 0} \times \mathbf{1 0}^{3}$ & $200 \times 10^{3}$ \\
$t_{i}^{0}$ & {$[\mathrm{MPa}]$} & 25 & $\mathbf{5 0}$ & 75 \\
\hline
\end{tabular}

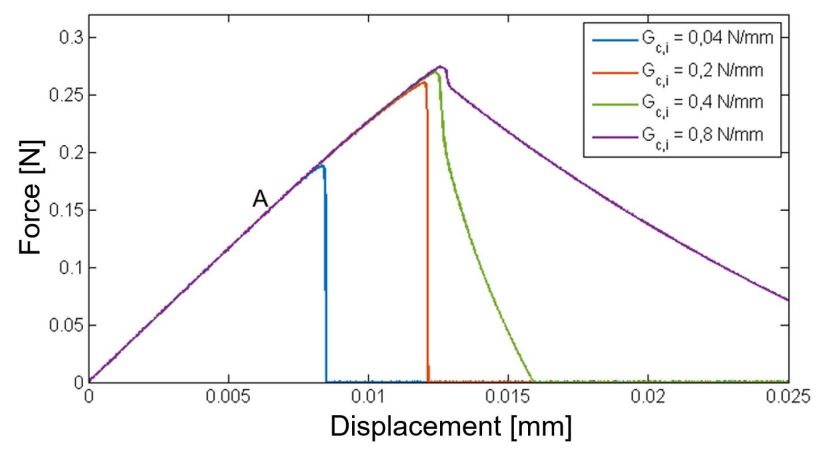

Figure 9 - Influence of the critical energy release rate on the force-displacement curve

ical energy release rate the same crack initiation point (A) is obtained, cf. Figure 9. The energy release rate $G_{\mathrm{c}, i}$ mainly influences the force maximum and the corresponding displacement, the point of complete debonding and the slope of the force drop. This is because the critical energy release rate controls the degradation process and the speed of the interface crack growth within the traction-separation law. For the critical energy release rate of $G_{\mathrm{c}, i}=0.04 \mathrm{~N} / \mathrm{mm}$, the force maximum is $F_{\max }=0.188 \mathrm{~N}$. After the force maximum, the load drop occurs abruptly. The load drop remains abrupt with increasing critical energy release rate up to $G_{\mathrm{c}, i}=0.2 \mathrm{~N} / \mathrm{mm}$. Here the force maximum is $38 \%$ higher than the previously for $G_{\mathrm{c}, i}=0.04 \mathrm{~N} / \mathrm{mm}$. For the critical energy release rate of $G_{\mathrm{c}, i}=0.4 \mathrm{~N} / \mathrm{mm}$, a load drop with a smaller gradient can be detected. With further increasing of $G_{\mathrm{c}, i}$ to $0.8 \mathrm{~N} / \mathrm{mm}$ the gradient becomes even smaller. With this high critical energy release rate, the complete debonding no longer takes place within a global displacement of $25 \mu \mathrm{m}$. The force maximum increases by about $1 \%$ compared to $G_{\mathrm{c}, i}=0.4 \mathrm{~N} / \mathrm{mm}$. It seems as if a saturation is reached above a certain critical energy release rate, that higher values do not lead to an increase in the force maximum, but slow down the load drop.

\subsubsection{Interface strength}

In Figure 10 the influence of the interface strength $t_{i}^{0}$ on the force-displacement curve is shown. The critical interface strength defines the crack initiation. The smaller the critical interface strength, the smaller the force max-

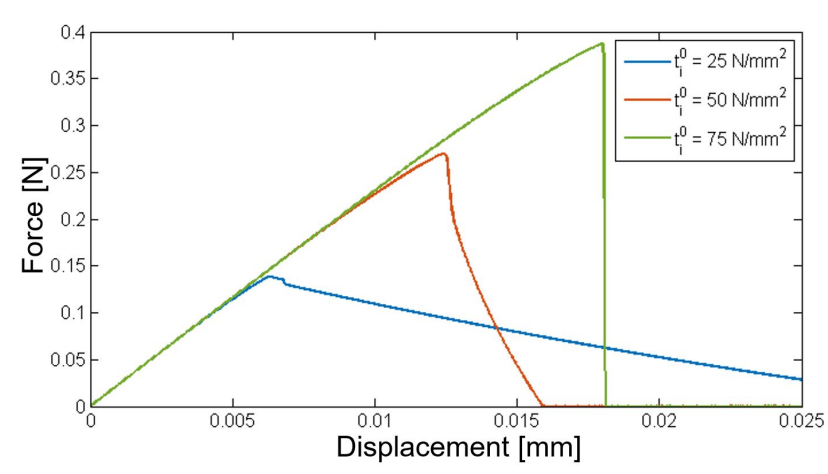

Figure 10 - Influence of the interface strength on the force-displacement curve

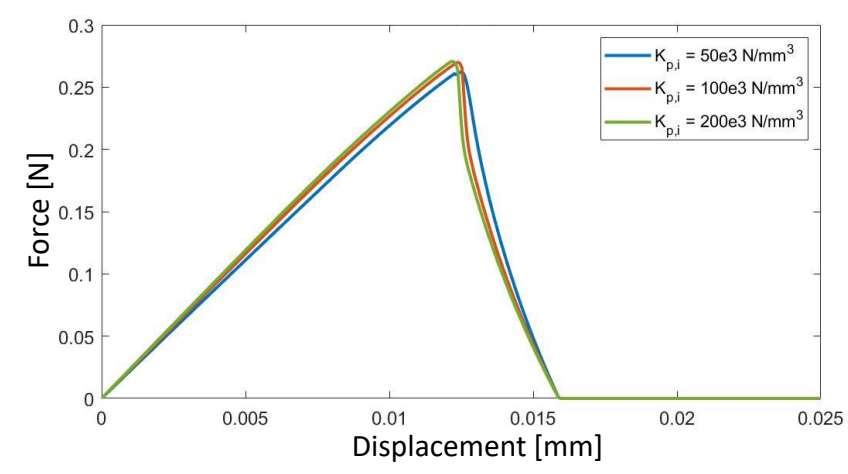

Figure 11 - Influence of the initial interface stiffness on the force-displacement curve

imum and the corresponding displacement. The critical interface strength has no influence on the initial gradient of the force-displacement curves, which remains identical until crack initiation. The gradients of the load drop are different because the interface strength changes the ratio of the portions of the critical energy release rate before and after crack initiation.

\subsubsection{Initial interface stiffness}

Figure 11 shows the force-displacement curves for three initial interface stiffnesses $K_{\mathrm{p}, i}$ of $50 \times 10^{3} \mathrm{~N} / \mathrm{mm}^{3}$, $100 \times 10^{3} \mathrm{~N} / \mathrm{mm}^{3}$ and $200 \times 10^{3} \mathrm{~N} / \mathrm{mm}^{3}$. The initial interface stiffness influences several factors in the force-displacement curve: The higher the initial interface stiffness, the higher is the initial slope of the force-displacement curve. This in turn increases the maximum force and decreases the corresponding displacement. As the initial interface stiffness increases but the interface strength $t_{i}^{0}$ is kept constant, crack initiation occurs at a smaller critical displacement $\delta_{i}^{0}$.

The percentage deviations from the simulation using the reference value of $K_{\mathrm{p}, i}=100 \times 10^{3} \mathrm{~N} / \mathrm{mm}^{3}$ are listed in Table 3. The global displacement at crack initiation is mostly influenced by the initial interface stiffness. The difference to the reference simulation is $31.5 \%$ for 
$K_{\mathrm{p}, i}=50 \times 10^{3} \mathrm{~N} / \mathrm{mm}^{3}$ and $28.4 \%$ for $K_{\mathrm{p}, i}=200 \times 10^{3} \mathrm{~N} / \mathrm{mm}^{3}$. For the other characteristic values, the deviation is less than $5 \%$. It can therefore be assumed that the initial interface stiffness has a small influence on these characteristic values. In addition, the changes in the initial slope becomes smaller as the initial interface stiffness increases.

\subsection{Calibration variants}

The parametric study reveals a complex interaction of the influences of the different interface parameters on the global force-displacement curve. Thus, the interface parameters cannot be derived directly from a specific characteristic value of the curve. Therefore, three methods are presented in the following which could be used to determine the interface parameters from forcedisplacement curves obtained in single fiber pull-out tests.

There are four model parameters to be defined by the calibration: The first is the unknown geometry parameter $l_{\text {fr }}$ (free fiber length) and the others are the tractionseparation law parameter of Mode II: The energy release rate $G_{\mathrm{c}, \mathrm{II}}$, the critical interface strength $t_{\mathrm{II}}{ }^{0}$ and the initial interface stiffness $K_{\mathrm{p}, \mathrm{II}}$. These parameters have the greatest influence on the force-displacement curve. The remaining mode-dependent traction-separation law parameters are defined by the following ratios, which are assumed based on literature data [2, 16, 20, 22], cf. Table 5 . In addition, the thermal prestress induced by a temperature difference of $\Delta T=-103 \mathrm{~K}$ is taken into account.

The aim of the calibration is to depict the characteristic quantities $F_{\mathrm{d}}, u_{\mathrm{A}}$ and $F_{\text {max }}$ from the reference experiment by [30], see Table 6 . The three calibration variants are described in the following.

\subsubsection{Variant $A$}

The procedure for calibration Variant A is shown in Figure 12. Four steps are used to determine the unknown parameters $l_{\mathrm{fr}}, K_{\mathrm{p}, \mathrm{II}}, t_{\mathrm{II}^{0}}$ and $G_{\mathrm{c}, \mathrm{II}}$. All four parameters are determined using the analytical model of Zhandarov et al. [29] and the analytical equations from [15, 28, 31].

In the first step the free fiber length $l_{\mathrm{fr}}$ is calculated. For this purpose,

$l_{\mathrm{fr}}=\frac{\pi r_{\mathrm{f}}^{2} E_{\mathrm{f}}}{F_{\mathrm{d}}}\left(u_{\mathrm{A}}-\frac{F_{\mathrm{d}}}{\pi r_{\mathrm{f}}^{2} E_{\mathrm{f}}} \tanh \left(\frac{\beta l_{\mathrm{e}}}{2}\right)-\frac{F_{\mathrm{d}}}{\pi r_{\mathrm{m}}^{2} E_{\mathrm{m}}}\left(l_{\mathrm{m}}-l_{\mathrm{e}}\right)\right)$

developed by Zhandarov et al. [29] is used. Herein, $E_{\mathrm{f}}$ and $E_{\mathrm{m}}$ are the Young's moduli of the fiber and the matrix, $r_{\mathrm{f}}$ and $r_{\mathrm{m}}$ are the radius of the fiber and the matrix,

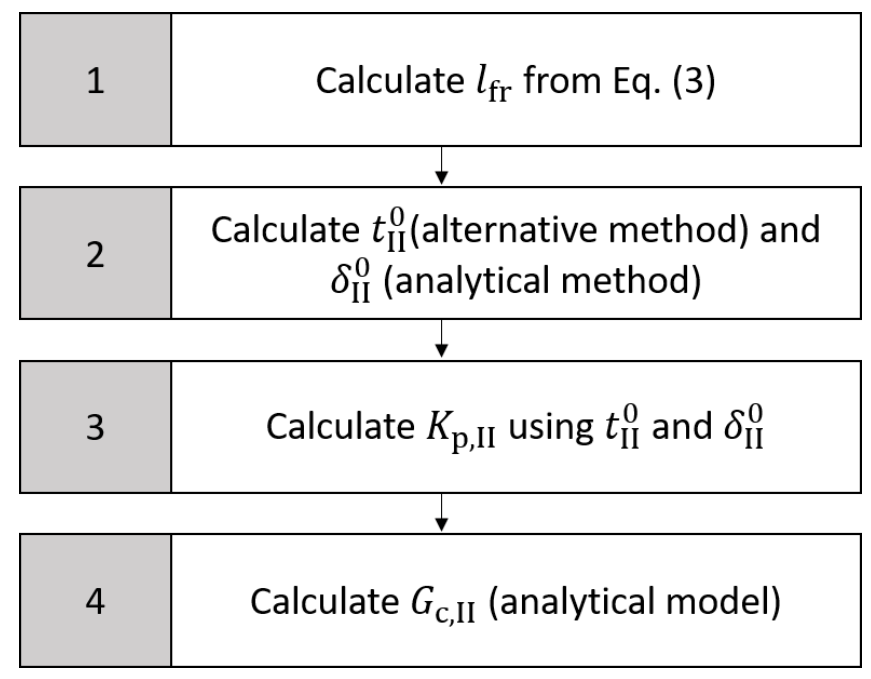

Figure 12 - Calibration Variant A

$l_{\mathrm{m}}$ is the length of the matrix in the real experiment, $l_{\mathrm{e}}$ is the embedded length of the fiber and $F_{\mathrm{d}}$ is the force when debonding starts and $u_{\mathrm{A}}$ is the corresponding displacement. Please note that $l_{\mathrm{m}}$ differs from $l_{\mathrm{x}}$, because the FE model is built up with reduced dimensions. Evaluating Equation (3) results in $l_{\mathrm{fr}}=0.511 \mathrm{~mm}$. To use the cohesive zone model, the initial interface stiffness

$$
K_{\mathrm{p}, \mathrm{II}}=\frac{t_{\mathrm{II}}^{0}}{\delta_{\mathrm{II}}^{0}}
$$

can be determined with the traction-separation law.

To use Equation (4), the critical interface strength $t_{\mathrm{II}}^{0}$ is calculated with the alternative method by $[30,31]$ and $\delta_{\text {II }}^{0}$ is obtained using the analytical model by [29]. The parameter $\delta_{\mathrm{II}}^{0}$ indicates the relative displacement of the contact surfaces of fiber and matrix. This corresponds to the critical displacement

$$
\delta_{\mathrm{II}}^{0}=\frac{P_{\mathrm{d}}}{\pi r_{\mathrm{f}}^{2} E_{\mathrm{f}}} \tanh \left(\frac{\beta l_{\mathrm{e}}}{2}\right)
$$

of the interface up to Point A [29]. The obtained values are $t_{\mathrm{II}}^{0}=100.35 \mathrm{MPa}$ and $\delta_{\mathrm{II}}^{0}=0.835 \mu \mathrm{m}$. This yields $K_{\mathrm{p}, \mathrm{s}}=120000 \mathrm{~N} / \mathrm{mm}^{3}$. In the fourth step the calculation of $G_{\mathrm{c}, \mathrm{II}}=0.6 \mathrm{~N} / \mathrm{mm}$ follows according to [29].

\subsubsection{Variant $B$}

Figure 13 shows the procedure for calibration Variant B. In the first step, the interface stiffness $K_{\mathrm{p}, \mathrm{II}}$ is calculated as in Variant A using the analytical equations and thus $K_{\mathrm{p}, \mathrm{II}}=120000 \mathrm{~N} / \mathrm{mm}^{3}$. In the second step, the free fiber length $l_{\mathrm{fr}}$ is now adjusted to force the forcedisplacement curve to run through Point $\mathrm{A}$ at $\left(u_{\mathrm{A}}, F_{\mathrm{d}}\right)$. In the third step, $t_{\mathrm{II}}^{0}$ is set so that crack initiation takes place at Point A at $\left(u_{\mathrm{A}}, F_{\mathrm{d}}\right)$. In the fourth step $G_{\mathrm{c}, \mathrm{II}}$ is 
Table 4 - Characteristic values of force-displacement curve obtained for different initial interface stiffnesses compared to the reference simulation with $K_{\mathrm{p}, i}=100 \times 10^{3} \mathrm{~N} / \mathrm{mm}^{3}$

\begin{tabular}{lccc}
\hline & Reference simulation with & \multicolumn{2}{c}{ Relative deviation to reference for } \\
& $K_{\mathrm{p}, i}=100 \times 10^{3} \mathrm{~N} / \mathrm{mm}^{3}$ & $K_{\mathrm{p}, i}=50 \times 10^{3} \mathrm{~N} / \mathrm{mm}^{3}$ & $K_{\mathrm{p}, i}=200 \times 10^{3} \mathrm{~N} / \mathrm{mm}^{3}$ \\
\hline Initial gradient of curve & $23.3 \mathrm{~N} / \mathrm{mm}$ & $-4.3 \%$ & $2.5 \%$ \\
Crack initiation (displacement) & $6.2 \mu \mathrm{m}$ & $31.5 \%$ & $-28.4 \%$ \\
Maximum force & $0.27 \mathrm{~N}$ & $-2.8 \%$ & $0.3 \%$ \\
Displacement at maximum force & $12.4 \mu \mathrm{m}$ & $1.2 \%$ & $-1.6 \%$ \\
\hline
\end{tabular}

Table 5 - Assumed relations between mode dependent traction-separation law parameters

\begin{tabular}{cc}
\hline Normal fracture mode & shear fracture modes \\
\hline$G_{\mathrm{c}, \mathrm{I}}=1 / 25 \cdot G_{\mathrm{c}, \mathrm{II}}$ & $G_{\mathrm{c}, \mathrm{III}}=G_{\mathrm{c}, \mathrm{II}}$ \\
$t_{\mathrm{I}^{0}}=2 / 3 \cdot t_{\mathrm{II}}{ }_{\mathrm{III}}=t_{\mathrm{II}}$ \\
$K_{\mathrm{p}, \mathrm{I}}=K_{\mathrm{p}, \mathrm{II}}=K_{\mathrm{p}, \mathrm{III}}$ \\
\hline
\end{tabular}

Table 6 - Characteristic values for calibration

\begin{tabular}{lc}
\hline Characteristic & Value \\
\hline$F_{\mathrm{d}}$ & $0.1875 \mathrm{~N}$ \\
$u_{\mathrm{A}}$ & $0.01 \mathrm{~mm}$ \\
$F_{\max }$ & $0.2487 \mathrm{~N}$ \\
\hline
\end{tabular}

modified in order to give a simulated maximum force $F_{\max }^{\text {sim }}$ which is equal to the maximum force of the experiment $F_{\max }^{\exp }$. Calibration Variant B yields $l_{\mathrm{fr}}=0.47 \mathrm{~mm}$, $t_{\mathrm{II}}^{0}=118 \mathrm{MPa}$ and $G_{\mathrm{c}, \mathrm{II}}=0.1 \mathrm{~N} / \mathrm{mm}$.

\subsubsection{Variant C}

In the last variant, the model is calibrated without the need for analytical models and equations. For this purpose, the coefficient $\gamma$ is introduced, which indicates the proportion of the complete critical energy release rate in relation to the energy release rate that has to be applied for the crack initiation. In this variant $\gamma$ is set to a fixed value so that $G_{\mathrm{c}, \mathrm{II}}$ can be calculated directly with $K_{\mathrm{p}, \mathrm{II}}$ and $t_{\mathrm{II}}^{0}$. The parameter $\gamma$ is a free parameter that has to be set in the calibration process. Physically, the parameter must be greater than 1, otherwise the energy release rate dissipated at crack initiation is higher than the whole critical energy release rate and no energy is available to dissipate during the damage propagation process. Since there is an abrupt load drop after Point $B$ in the reference experiment, the value should not be too large. Therefore, a value of $\gamma=2$ is chosen. This is comparable to the value obtained by Variant $\mathrm{B}$, where $\gamma=1.75$. Figure 14 shows the procedure for Variant C.

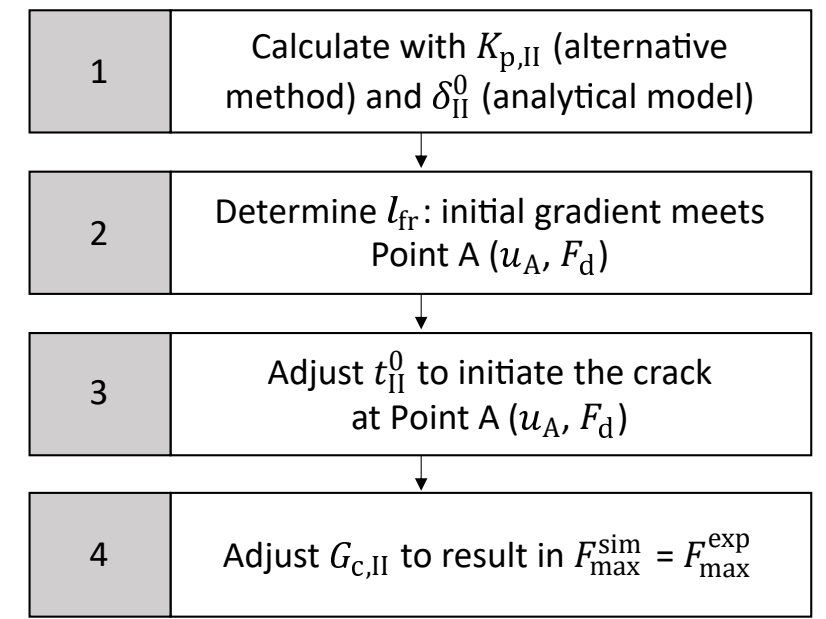

Figure 13 - Calibration Variant B

To determine the model parameters, an iterative procedure is necessary. In the first step $K_{\mathrm{p}, \mathrm{II}}$ is estimated. In step two, the free fiber length $l_{\mathrm{fr}}$ is adjusted as described in Variant B. Now, analogous to Variant B, $t_{\mathrm{II}}^{0}$ is set so that the crack initiates at the displacement $u_{\mathrm{A}}$. At the same time the critical energy release rate

$$
G_{\mathrm{c}, \mathrm{II}}=\frac{\gamma\left(t_{\mathrm{II}}^{0}\right)^{2}}{2 K_{\mathrm{p}, \mathrm{II}}}
$$

is calculated as a function of $t_{\mathrm{II}}^{0}, K_{\mathrm{p}, \mathrm{II}}$ and $\gamma$. Then $F_{\max }$ is checked as a second criterion by comparing the simulated value $F_{\max }^{\mathrm{sim}}$ with the value from the experiment $F_{\max }^{\exp }$. If $F_{\max }^{\mathrm{sim}}$ is too large, $K_{\mathrm{p}, \mathrm{II}}$ is decreased; if $F_{\max }^{\mathrm{sim}}$ is too small, $K_{\mathrm{p}, \mathrm{II}}$ is increased. The steps are repeated iteratively until $F_{\max }^{\text {sim }}$ and $F_{\text {max,Exp }}^{\exp }$ are equal within a given tolerance of $\varepsilon \leq 2 \%$. The calibration by Variant C yield $K_{\mathrm{p}, \mathrm{s}}=85000 \frac{\mathrm{N}}{\mathrm{mm}^{3}}, l_{\mathrm{fr}}=0.466 \mathrm{~mm}, t_{\mathrm{s}}^{0}=95.5 \mathrm{MPa}$ and $G_{\mathrm{c}, \mathrm{II}}=0.107 \frac{\mathrm{N}}{\mathrm{mm}}$.

\subsubsection{Comparison of the calibration variants}

Table 7 shows the calibrated parameters and the characteristic values of the calculated force-displacement curve of the three variants. It can be seen that Variants 


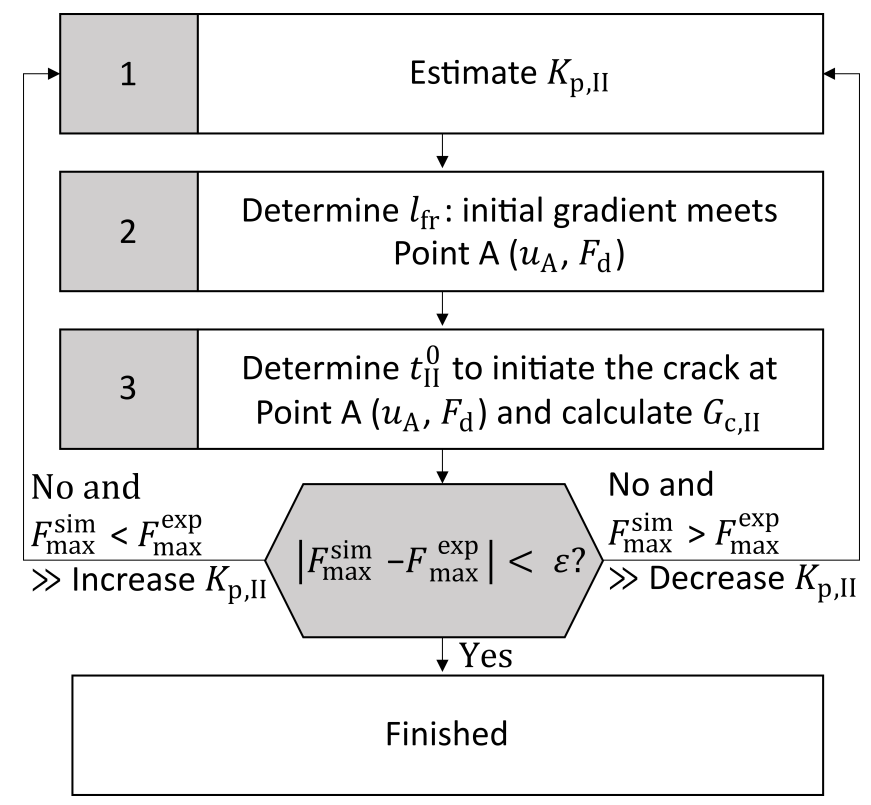

Figure 14 - Calibration Variant C

$\mathrm{B}$ and $\mathrm{C}$ deliver similar parameters and that the characteristic quantities correspond to the values from the experiment, see Table 6. In Variant A, in which the parameters are calculated from the analytical equations, the characteristic quantities do not match those from the force-displacement curve from the experiment. This can be explained the different assumptions of the analytical models used for calibration, e.g., for the calculation of the thermal prestresses or for the consideration of the interface friction force. Additionally, the interface parameters $t_{\mathrm{II}}^{0}$ and $G_{\mathrm{c}, \mathrm{II}}$ are calculated by the analytical models with consideration of the interface friction, which is not captured by the cohesive zone model. This is also the reason for the lower values of $t_{\mathrm{II}}^{0}$ and $G_{\mathrm{c}, \mathrm{II}}$ for Variant A compared to Variant B and C. The parameters of the latter are calibrated directly to fit the experimental characteristic values of Table 6 and therefore incorpo-

Table 7 - Resulting model parameters and characteristic values from calibration Variants A, B and C in comparison

\begin{tabular}{llccc}
\hline & & Variant A & Variant B & Variant C \\
\hline$l_{\mathrm{fr}}$ & {$[\mathrm{mm}]$} & 0.551 & 0.47 & 0.466 \\
$K_{\mathrm{p}, \mathrm{II}}$ & {$\left[\mathrm{N} / \mathrm{mm}^{3}\right]$} & $120 \times 10^{3}$ & $120 \times 10^{3}$ & $85 \times 10^{3}$ \\
$t_{\mathrm{II}}$ & {$[\mathrm{MPa}]$} & 100 & 118 & 95.5 \\
$G_{\mathrm{c}, \mathrm{II}}$ & {$[\mathrm{N} / \mathrm{mm}]$} & 0.06 & 0.1 & 0.1073 \\
$\gamma$ & {$[-]$} & 1.44 & 1.72 & 2 \\
\hline$u_{\mathrm{A}}$ & {$[\mathrm{mm}]$} & 0.0083 & 0.01 & 0.01 \\
$F_{\mathrm{d}}$ & {$[\mathrm{N}]$} & 0.1468 & 0.1874 & 0.18745 \\
$F_{\max }$ & {$[\mathrm{N}]$} & 0.1752 & 0.2489 & 0.2485 \\
\hline
\end{tabular}

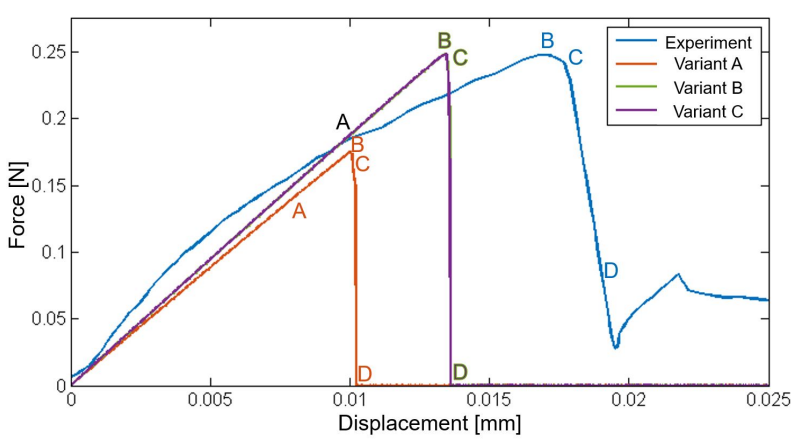

Figure 15 - Comparison of the calibration variants with the experiment

rate the frictional effect in an indirect manner. Hence, only the Variants B and C are suitable for the calibration of the cohesive zone model. For these variants, one of the parameters to be calibrated must be specified in advance to determine the other parameters. This does not result in a unique solution for calibration. Comparing Variant $\mathrm{B}$ and $\mathrm{C}$, Variant $\mathrm{C}$ is more time consuming than Variant $\mathrm{B}$. An advantage of Variant $\mathrm{C}$ is that no analytical parameter has to be used and the parameters can be directly fitted to the experimental characteristics without additional assumptions.

\subsection{Comparison of FE model to the reference experiment}

Figure 15 shows the force-displacement curves of the experiment and the calibration Variants A, B and C of the FE model. It can be seen that the maximum force as well as the crack initialization point of the force-displacement curves of Variant $B$ and $C$ correspond with the experiment. For Variant A, both the initial gradient up to Point $\mathrm{A}$, as well as the displacement at the force maximum and the force maximum itself are less accurate compared to the other variants. The estimates from the analytical models can therefore not be readily adopted to the cohesive zone approach. The curves of the forcedisplacement diagram of all three variants differ from the experiment. The first range up to the crack initialization Point A is linear in the FE model. In the experiment, this shows a non-linearity. One reason could be the idealized matrix form which is used in the FE model and therefore the range up to Point A cannot be mapped exactly $[25,26]$. Another reason could be material nonlinearities, which can occur under high deformation in the matrix material and are not considered [29]. The displacement at the force maximum is about $24 \%$ smaller in the FE model of Variant B and C than in the experiment. This can be explained by the fact that the interface behavior is not accurately represented with the tractionseparation law. In order to reduce the slope of the curve 
in the section from Point A to B compared to the section from $\mathrm{O}$ to $\mathrm{A}$, a faster crack growth should be mapped in the traction-separation law. At the same time, an interface friction force would have to counteract the crack growth, resulting in a higher displacement at the force maximum. This phenomenon cannot be depicted by the traction-separation law, because the degradation behavior does not include a constant friction force. This can also be seen when the fiber is completely separated from the matrix. At that point the load drops to $0 \mathrm{~N}$. A frictional force must be modeled here as well. For a more precise representation of the force-displacement behavior, the interface friction force should therefore be taken into account in the traction-separation law.

\section{Peridynamic simulation}

\subsection{Failure in peridynamics}

In the following the pull out analysis with a peridynamic model will be presented. Due to the formulation the hope is that a similarly good solution can be achieved with fewer parameters compared to the continuum mechanical solution. Peridynamics describe a non local continuum model. Each material point is influenced by all other material points that are located in its horizon radius [18]. The interaction of the material points is defined by bonds. To determine if damage occurs, a criterion has to be used to evaluate the bond behavior locally. Foster et al. [7] and Willberg et al. [23] derived a maximum elastic bond potential value $w_{\mathrm{c}}$. The elastic bond for the three-dimensional

$$
w_{\mathrm{c}}=\frac{4 G_{0}}{\pi \delta^{4}}
$$

and for the two-dimensional case

$$
w_{\mathrm{c}}=\frac{3 G_{0}}{\pi \delta^{3} h}
$$

depends on the energy release rate $G_{0}$ and the horizon $\delta$ For the two-dimensional case, the bond potential value depends on the thickness $h$, too. If $w_{\mathrm{c}}$ exceeds a critical value, damage initiates due to a bond failure. If the critical strain is reached, the bond is released [19]. For tracing the crack, a damage variable is used which is calculated by dividing the number of released bonds of a material point by the total number of initial bonds of this point.

\subsection{Peridynamic model}

\subsubsection{Material}

The material data is taken from Table 1. An isotropic linear-elastic material model is used for the fiber and

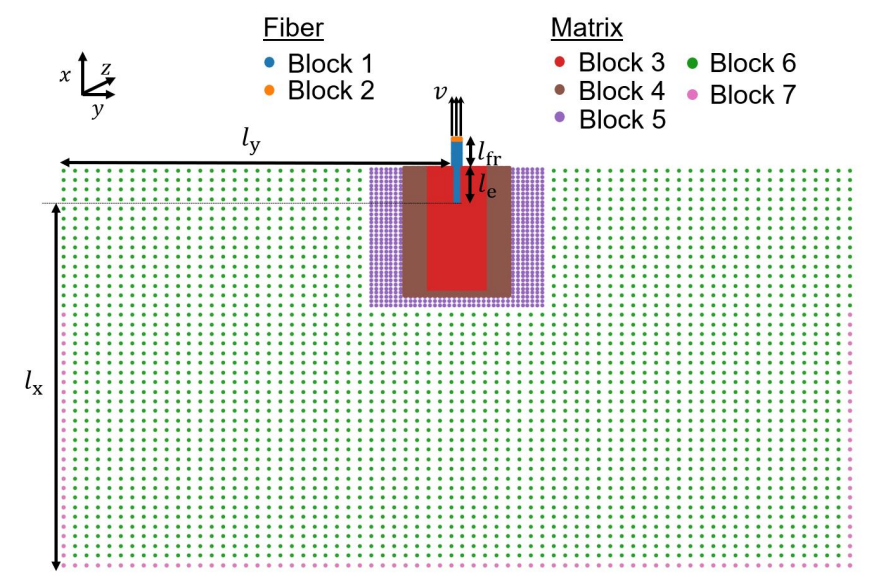

Figure 16 - Peridynamic model

matrix. Comparative values from the literature are used for the density. The density of the Araldite L556 epoxy resin is about $1.2 \mathrm{~g} / \mathrm{cm}^{3}$ [10]. For glass fiber a density of $2.56 \mathrm{~g} / \mathrm{cm}^{3}[5]$ is used.

\subsubsection{Model geometry and boundary conditions}

The peridynamic model is shown in Figure 16. The structure is divided into fiber and matrix. In peridynamics, three-dimensional models are generated. This model is two-dimensional with a row of points in $z$ direction. This simplification is assumed, because a complete three-dimensional description of the fiber and matrix would lead to a high computational effort, because the number of material points would be significantly higher compared to the two-dimensional simplification. The possibility of an axisymmetric model is not yet implemented in the software. A further possibility would be the symmetrical view of the model. This is not used because the influence of the boundary conditions should be avoided. In order to consider the influence of rotational symmetry, the volume of the elements is increased with increasing distance from the symmetry line of the model. The volume for an element is

$$
V_{\mathrm{R}}=d x^{2}|R| \pi,
$$

where $d x$ is the distance between the material points and $R$ is the radius of the axisymmetric model. In Figure 17, the adaptation of the volume is shown qualitatively.

The length of the fiber $l_{\text {ges }}$, the free fiber length $l_{\text {fr }}$ and the embedded fiber length $l_{\mathrm{e}}$, are reduced compared to the reference experiment in order to reduce the computational effort and to shorten the computational time until the crack is detected. Due to the elastic strain of the fiber, the global displacement required for crack initialization becomes larger, the longer the free fiber length is. By reducing the embedded fiber length, the number of material points in the fiber and matrix and 


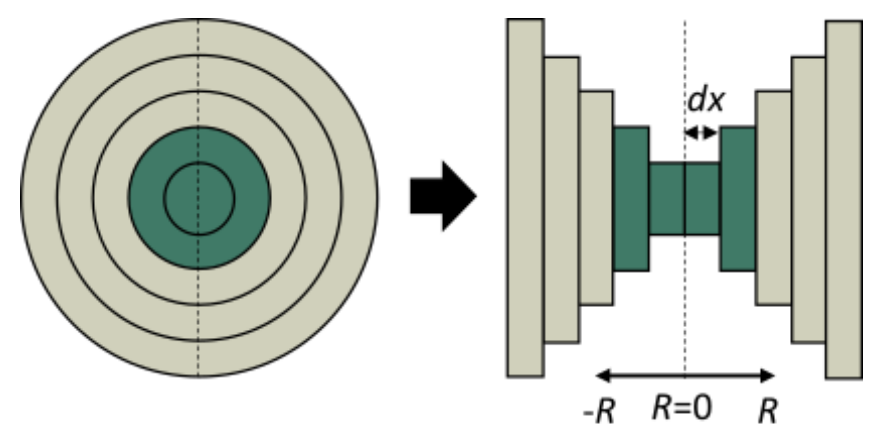

Fiber Matrix Symmetry line

Figure 17 - Adaptation of the axisymmetric volume

the global displacement can be reduced. For the free fiber length, $l_{\mathrm{fr}}=50 \mu \mathrm{m}$ is defined. With $l_{\mathrm{e}}=60 \mu \mathrm{m}$ the embedded fiber length is $47 \%$ of the embedded fiber length in the reference experiment. For the matrix dimensions the lengths $l_{\mathrm{x}}=532 \mu \mathrm{m}$ and $l_{\mathrm{y}}=464 \mu \mathrm{m}$ are assumed. The matrix dimensions are chosen larger compared to the FE model, because the boundary effects due to the clamping have a stronger influence on the deformation at the fiber-matrix interface. The matrix dimensions are increased to such an extent that no influence of the boundary can be detected. In addition, the material points are arranged symmetrically, since asymmetry could have side effects on the calculation.

The fiber is shown with Blocks 1 and 2 in Figure 16. At Block 2 the load is defined. A load velocity $v$ in positive $x$-direction is applied, which has a value of $v=0.1 \mathrm{~mm} / \mathrm{s}$. The load velocity is about 100 times higher than the load velocities used in the real experiment [29]. The velocity is selected higher in order to reduce the calculation effort for the same end displacement applied. The consequence of a higher speed is a greater inaccuracy, because a higher force impulse acts on the system in a shorter time and thus results in a higher oscillation amplitude in the force response. The influence of a higher speed should be investigated in further studies.

The matrix is divided into Blocks 3-7. Block 7 represents the bearing which prevents displacement in $x$ and $y$-direction. Since the dimensions of the model are close to the real size of the matrix diameter of $1.25 \mathrm{~mm}$, the bearing is only applied in the lower part of the matrix. The Blocks 3-6 differ in the distance between the material points, the horizon radius and the state of damage. In Blocks 1-2 the distance of the material points is the same. In Blocks 5-7, the distance between the material points is increased to reduce the calculation effort. Since the crack is expected in Block 3 , the damage is only activated there. No damage is assumed in the remaining blocks to reduce the numerical cost by running the damage evaluation routine. Also for the connected

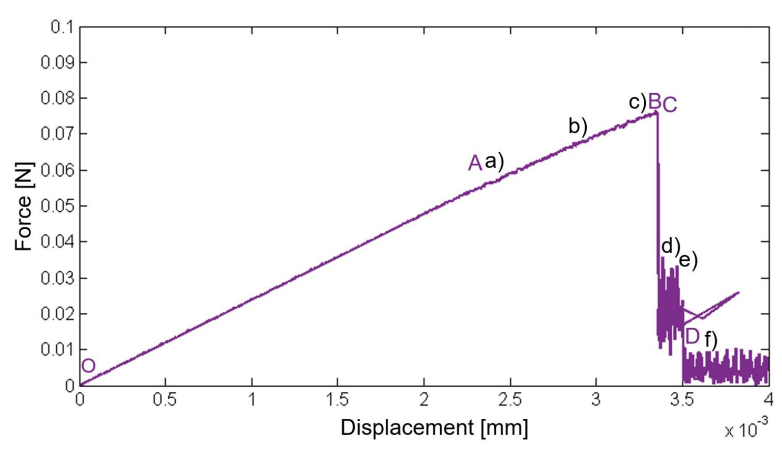

Figure 18 - Force-displacement diagram of the peridynamic model with crack status

fiber model in Block 2 no damage model is activated. This is because the critical energy release rate evaluated in this region corresponds to the fiber. Therefore, it is drastically larger compared to the matrix material and no bond failure is expected. This results in a total number of 9572 material points. An overview of the blocks and a description is shown in Table 8.

An explicit solution algorithm is used for the calculation. To reduce the simulation time, a mass scaling of $10^{3}$ is applied.

\subsection{Cracking}

In this section, the simulated crack process of the peridynamic model is presented. Six different points in time (a-f) are selected from the calculation, which represent the crack propagation in the structure. The points of time are assigned to the force-displacement curve in Figure 18, and Figure 19 shows the visualization of the crack propagation using the damage variable.

The crack initiation at Point A can be recognized in the force-displacement curve by the fact that the system response has a higher oscillation amplitude than the previous curve. The crack initiation starts at the Point $x=0$. It can be seen that the crack starts three material points next to the fiber edge. In addition, damage occurs

Table 8 - Properties of the model blocks

\begin{tabular}{cccl}
\hline Block & Part & $\begin{array}{l}\text { Distance } \\
\text { of points }\end{array}$ & $\begin{array}{l}\text { Horizon } \\
\text { radius }\end{array}$ \\
\hline 1 & Fiber & $2.223 \mu \mathrm{m}$ & $8.915 \mu \mathrm{m}$ \\
2 & Fiber & $2.223 \mu \mathrm{m}$ & $8.915 \mu \mathrm{m}$ \\
3 & Matrix & $2.223 \mu \mathrm{m}$ & $8.915 \mu \mathrm{m}$ \\
3 & Matrix & $2.223 \mu \mathrm{m}$ & $8.915 \mu \mathrm{m}$ \\
4 & Matrix & $2.223 \mu \mathrm{m}$ & $8.915 \mu \mathrm{m}$ \\
5 & Matrix & $6.669 \mu \mathrm{m}$ & $26.74 \mu \mathrm{m}$ \\
6 & Matrix & $15.56 \mu \mathrm{m}$ & $62.40 \mu \mathrm{m}$ \\
7 & Matrix & $15.56 \mu \mathrm{m}$ & $62.40 \mu \mathrm{m}$ \\
\hline
\end{tabular}




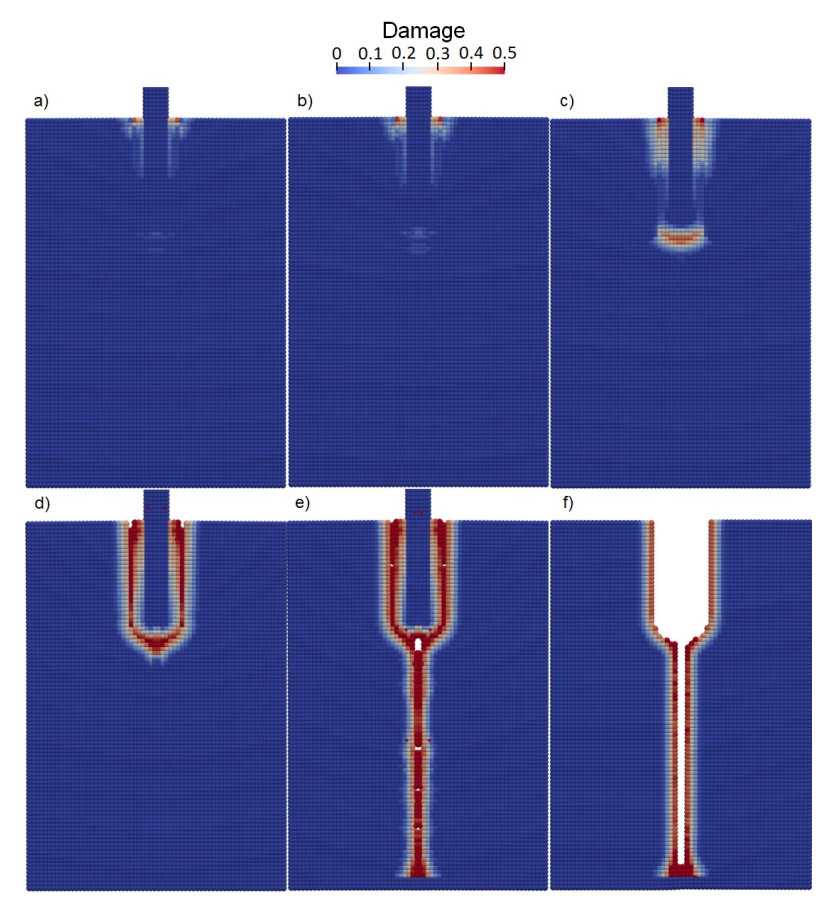

Figure 19 - Cracking status of the peridynamic simulation

below the fiber bottom in the matrix, see Figure 19b-c. Both initiation points are Mode I dominated. The results correspond to the crack formation process of the FE model and the experimental findings. The damage index in Peridynamics differs from that used in the finite element model. It is defined as the lost bonds of a material point in relation to all its original bonds. Therefore, an index of 0.5 means that $50 \%$ of all bonds are lost. The point in time shown in Figure 19c is directly before the load drop in Figure 18c. The load drop is caused by an unstable crack growth which is shown in Figure 18d-f. The crack propagation is Mode II dominated. First, the crack grows parallel on the left and right side of the matrix half and then joins the crack below the fiber ground, see Figure 19d. Then the crack continues to develop below the fiber and runs along the axis of symmetry, cf. Figure 19e. The crack growth leads up to Block 4, where the damage is deactivated. The crack in the axis of symmetry seems to be favored by the unstable crack propagation and the low volume in the axis of symmetry. Due to the adjusted volume because of the rotational symmetry, see Figure 17 , the smallest volume is located in the axis of symmetry. Therefore, this is the critical point at which crack propagation occurs. This is followed by the complete separation of the fiber from the matrix, cf. Figure 19f. In this figure the fiber is no longer present in this image area, due to the scaling of the deformation. It must be noted that the vertically crack growth in the center of the model occurs, because no axisymmetric model was used. This leads to an over-
Table 9 - Parametric study of the model parameter

\begin{tabular}{lllll}
\hline Parameters & & \multicolumn{4}{c}{ Variation } \\
\hline $\begin{array}{l}\text { Critical energy } \\
\text { release rate }\end{array}$ & {$[\mathrm{N} / \mathrm{mm}]$} & 0.05 & $\mathbf{0 . 1}$ & 0.15 \\
$\begin{array}{l}\text { Horizon radius } \\
\text { Block 1-4 }\end{array}$ & {$[\mathrm{mm}]$} & $3 d x$ & $\mathbf{4 d x}$ & $5 d x$ \\
\hline
\end{tabular}

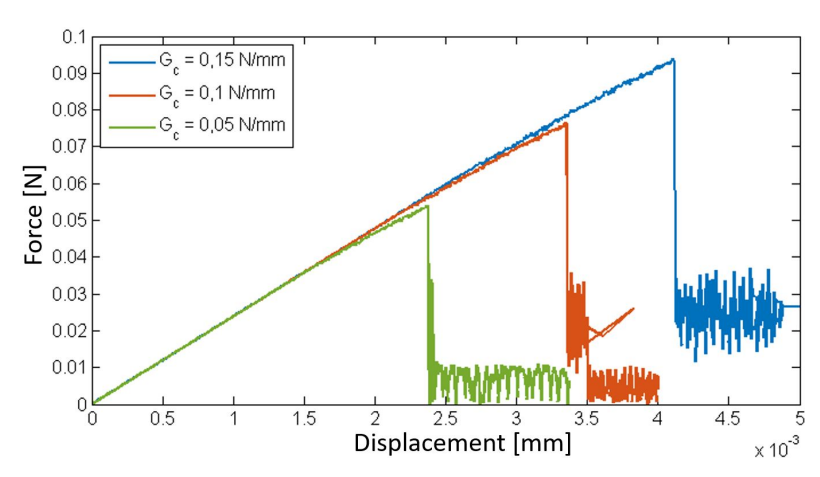

Figure 20 - Influence of the energy release rate on the force-displacement curve

estimation of bond energy and therefore to a failure of these bonds.

\subsection{Parameter study}

In this section the influence of the critical energy release rate $G_{\mathrm{c}}$ and the horizon radius $\delta$ on the forcedisplacement curve is examined. The range in which these parameters are varied is shown in Table 9. The bold numbers indicate the reference values of the parameters. The free fiber length $l_{\mathrm{fr}}$ is chosen to be $50 \mu \mathrm{m}$. The remaining material and model parameters are taken from Section 3.2.2.

\subsubsection{Critical energy release rate}

Figure 20 shows the influence of the critical energy release rate on the force-displacement curve. The initial slope of the curves is the same for different critical energy release rates, which can be seen from the fact that the curves overlap. The higher the critical energy release rate $G_{c}$, the greater the displacement at the force maximum and the greater the force maximum $F_{\text {max }}$ The maximum force at the force-displacement curve is $0.052 \mathrm{~N}$ with the parameter $G_{\mathrm{C}}=0.05 \mathrm{~N} / \mathrm{mm}$. If the critical energy release rate is doubled to $G_{\mathrm{c}}=0.1 \mathrm{~N} / \mathrm{mm}$, the maximum force $F_{\text {max }}$ increases by $44 \%$, if the critical energy release rate is tripled to $G_{\mathrm{c}}=0.15 \mathrm{~N} / \mathrm{mm}$, the maximum force $F_{\max }$ increases by $79 \%$. 


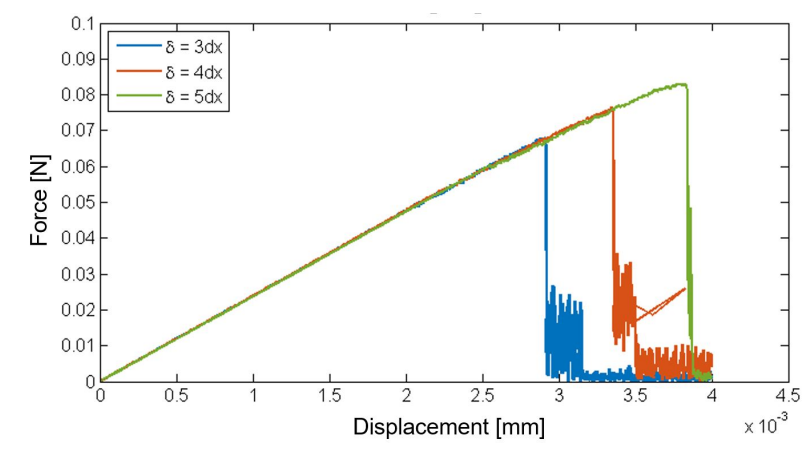

Figure 21 - Influence of the horizon radius on the forcedisplacement curve

\subsubsection{Horizon radius}

Besides the discretization, the horizon radius has an influence on the peridynamic solution. Figure 21 shows the influence of the horizon radius on the force-displacement curve. It shows that with a larger horizon radius the displacement of the force maximum and the force maximum itself becomes larger. This leads to a larger displacement at the force maximum. This means that the problem is not fully converged, besides the fact that the initial gradient with different horizon radius remains the same. If the horizon is reduced to zero and the material points are increased to infinity the model is not valid as it is for classical continuum mechanical models. Therefore, for convergence the initiation of the crack is chosen as measure. If this value changes marginally the model is assumed converged, because there is no underlying analytical description of this type of crack propagation problem. Compared to the reference parameter of $\delta=4 d x$ leading to a maximum force of $F_{\max }=0.074 \mathrm{~N}$, the maximum force increases by $12 \%$ using $\delta=5 d x$ and decreases by $7 \%$ with $\delta=3 d x$.

\subsection{Comparison of peridynamic model to FE model}

A calibration of the peridynamics model with the experiment is not possible. Here, an adjustment of the free fiber length would be necessary to reach the necessary end displacement of the experiment. The simulation of the experimental end displacement by means of the peridynamic model is connected with a high computational effort. This is why it is not included in this paper. Instead, the peridynamic model is compared with the FE model. For the comparison of the FE model and the peridynamic model the same dimensions for the free fiber length with $l_{\mathrm{fr}}=50 \mu \mathrm{m}$ and the embedded fiber length $l_{\mathrm{e}}=60 \mu \mathrm{m}$ are chosen, which are also used in the peridynamic calculations in Section 3.2.2. The matrix dimensions are carried out model-specific, as described

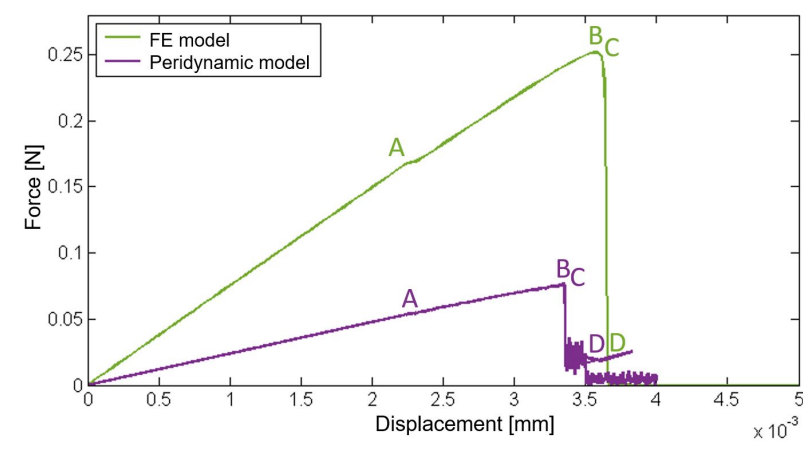

Figure 22 - Comparison of the peridynamic model and the FE model

in the respective modeling. The matrix dimensions are not chosen to be the same, because the boundary effects have a different influence on the displacement in the different models.

Figure 22 compares the force-displacement curves of the two numerical models. The model parameters for the FE model are taken from the model calibration with Variant $B$. This results in an energy release rate of $G_{\mathrm{c}, \mathrm{II}}=0.1 \mathrm{~N} / \mathrm{mm}$ and a critical interface strength of $t_{\mathrm{II}}^{0}=118 \mathrm{MPa}$. The thermal prestressing is not taken into account, since it is also not considered in the peridynamic model. For the peridynamic model, the critical energy release rate is $G_{\mathrm{c}}=0.1 \mathrm{~N} / \mathrm{mm}$ and the horizon radius is set to $\delta=8.9156 \mu \mathrm{m}$.

One difference between the models is the influence of the displacement, which is visible in the boundary conditions. The peridynamic model requires a more than 17 times larger matrix dimension than the FE model. A second difference is the crack path, which is not aligned along the real interface line, but is located three material points away from the fiber in the matrix due to its nonlocal nature. On the contrary to the FE based model where the crack path is predefined in the peridynamic model the crack could occur everywhere. However, for denser discretization the crack path will be nearer to the interface. The crack propagation is similar in the FE model and the peridynamic model, see Section 3.3. In both models, the crack initiation starts at $x=0$. In the peridynamic simulation, the fiber bottom debonding takes place at an earlier displacement than the unstable crack growth. This is not the case with the FE model with an embedded fiber length of $l_{\mathrm{e}}=129 \mu \mathrm{m}$, where the fiber debonding occurs together with the unstable crack growth. However, using the embedded fiber length of $l_{\mathrm{e}}=60 \mu \mathrm{m}$ the crack occurs earlier, too, which can be seen by a discontinuity in the force-displacement curve. This is followed by the unstable crack growth in both models. The force-displacement curve of the FE model and the peridynamic model are qualitatively similar, see 
Figure 22. The range until crack initiation is linear for both models, after that the curve becomes slightly nonlinear. The force maximum is followed by an abrupt load drop down to a force close to $0 \mathrm{~N}$. The model curves differ quantitatively. The initial slope of the peridynamics simulation is about three times lower than the FE model. This is a result of the differently structured models. In the peridynamic model the axissymmetry can only be partially represented. The coupling of the model halves is neglected. This could be an explanation for lower stiffness of the model.

Because the peridynamic implementation is not yet so technically mature, there are large deviations from the FE solution generated with a commercial software. However, the simulations with the peridynamic model show the advantages of the method compared to the FE method: The number of model parameters needed is smaller, e.g. no initiation criterion is needed. Since the interface failure parameters are difficult to determine experimentally, Peridynamics offer the chance to support this numerically. The free crack propagation is in principle also realizable with fracture mechanical models, which are included within a FE software. In this case the computational times are comparable to a peridynamic modeling. The advantage of the peridynamic modeling presented here is that no additional models for direction of the crack propagation have to be calculated. Therefore, the failure patterns can be determined in advance for more complex load conditions. A cohesive zone based modeling does not offer this possibility, although there are concepts which implement cohesive zones between all element boundaries. In contrast, the direction of crack propagation is defined in advance, which is absolutely permissible for interface analyses and leads to significantly more efficient analyses. The peridynamic model was run with eight CPUs on a $2.9 \mathrm{GHz}$ Intel Xeon E5-2690 processor and needs eight hours for calculation. The solution of the FE model takes only 80 minutes on two CPUs.

Further investigations in this area are necessary to improve the numerical efficiency. Axisymmetric peridynamic models presented by Zhang and Qiao [32] and peridynamic cohesive zones [24] are two promising approaches to close the efficiency gap for this type of analysis.

\section{Conclusions}

Numerical simulations of the fiber-matrix debonding in single fiber pull-out tests were carried out with an FE model using the cohesive zone approach and a peridynamic model. The aim of the simulation was to deter- mine the interface parameters for the respective model. In the FE model, the traction-separation law parameters of Mode II and the free fiber length have the greatest influence on the simulated pull-out test results and thus on the force-displacement curves. Different calibration variants were developed, whereby for Variants B and C the characteristic points of the reference experiment are captured. However, there is no unique solution, because there are more unknown parameter than information for calibration. Since the interface friction force is not considered in the traction-separation law, it can be assumed that the critical energy release rate for Mode II is overestimated by the calibration Variants B and C.

In the peridynamics model, the energy release rate and the horizon radius play a decisive role in addition to the free fiber length. A calibration was not possible within the present study, because the computational effort for the model is too large. For a final calibration of the peridynamic model, the possibility of axissymmetric modeling has to be implemented in Peridigm to be able to reduce the model size and therefore the computational effort. This allows to consider the original fiber length used in the experiment which is an important aspect for parameter identification. Afterwards, convergence of the peridynamic solution regarding distance of material points and horizon radius has to be ensured which could not be achieved within this study. Using this further developed model, the critical energy release rate $G_{0}$ defined for initiation of bond release in Peridigm can be adapted in the calibration process.

Code Availability: The finite element analyses in the present paper have been performed using ABAQUS $\odot$ 2018. The source code of the software Peridigm used for the peridynamic simulations is available under

$$
\text { https://github.com/PeriDoX/PeriDoX }
$$

(BSD 3-Clause license).

Acknowledgements: This research was funded by the German Research Foundation, DFG, project number 379482657.

\section{References}

[1] ABAQUS Anaysis User's Manual. Contact cohesive behavior, 2018.

[2] M. L. Benzeggagh and M. Kenane. Measurement of mixedmode delamination fracture toughness of unidirectional glass/epoxy composites with mixed-mode bending apparatus. Composites Science and Technology, 1996.

[3] V. Bheemreddy, K. Chandrashekhara, L. R. Dharani, and G. E. Hilmas. Modeling of fiber pull-out in continuous fiber reinforced ceramic composites using finite element method and artificial neural networks. Computational Materials Science, 2013. 
[4] Lüders C., Sinapius M., and Krause D. Adaptive cycle jump and limits of degradation in micromechanical fatigue simulations of fibre-reinforced plastics. International Journal of Damage Mechanics, 28(10):1523-1555, 2019.

[5] Cuvalci H., Erbay K., and Ipek H. Investigation of the effect of glass fiber content on the mechanical properties of cast polyamide. Arabian Journal for Science and Engineering, 2014. ISSN 1319-8025.

[6] Jung-Jun Park Doo-Yeol Yoo and Sung-Wook Kim. Fiber pullout behavior of HPFRCC: Effects of matrix strength and fiber type. Composite Structures, pages 263-276, 2017.

[7] J.T. Foster, S.A. Silling, and W. Chen. An energy based failure criterion for use with peridynamic states. International Journal for Multiscale Computational Engineering, 9(6):675-688, 2011. ISSN 0020-7683.

[8] A. Hampe, G. Kalinka, S. Meretz, and E. Schulz. An advanced equipment for single-fibre pull-out test designed to monitor the fracture process. 1994.

[9] Edith Mäder Hanna M. Brodowsky, Wolfgang Jenschke. Characterization of interphase properties: Microfatigue of single fibre model composites. Composites: Part A, pages 1579-1586, 2010.

[10] I. Infanta Mary Priya and Vinayagam B. K. Enhancement of biaxial glass fibre reinforced polymer composite with graphene platelet nanopowder modifies epoxy resin. Advances in $\mathrm{Me}$ chanical Engineering, 2018.

[11] Y. Y. Jia, W. Yan, and H.-Y. Liu. Numerical study on carbon fibre pullout using a chohesive zone model. 18th International Conference on Composite Materials, 2011.

[12] R. Kerans and T. Parthasarathy. Theoretical analysis of the fiber pullout and pushout tests. Journal of the American Ceramic Society, 1991.

[13] C. Lüders. Nonlinear-elastic orthotropic material modeling of an epoxy-based polymer for predicting the material behavior of transversely loaded fiber-reinforced composites. Journal of Composites Science, 4(46), 2020.

[14] C. Marotzke. Influence of the fiber length on the stress transfer from glass and carbon fibers into a thermoplastic matrix in the pull-out test. Composite Interfaces, 1993

[15] J. A. Nairn. Analytical fracture mechanics analysis of the pullout test including the effects of friction and thermal stresses. Adv Compos Lett, 2000.

[16] S. Ogihara and J. Koyanagari. Investigation of combined stress state failure criterion for glass fiber/epoxy interface by the cruciform specimen method. Composites Science and Technology, 2009.

[17] L. S. Penn and E. R. Bowler. A new approach to surface energy characterization for adhesive performance prediction. Surface and Interface Analysis, 1981

[18] S. A. Silling. Reformulation of elasticity theory for discontinuities and long-range forces. Journal of the Mechanics and Physics of Solids, 48(1):175-209, 2000. ISSN 0022-5096.

[19] S. A. Silling and E. Askari. A meshfree method based on the peridynamic model of solid mechanics. Computers \& Structures, 83(17):1526-1535, 2005. ISSN 0045-7949.

[20] Q. Sun, Z. Meng, G. Zhou, S.-P. Lin, H. Kang, S. Keten, H. Guo, and X. Su. Multi-scale computational analysis of unidirectional carbon fiber reinforced polymer composites under various loading conditions. Composite Structures, 2018. ISSN 02638223.

[21] T. J. Vaughan and C. T. McCarthy. Micromechanical modelling of the transverse damage behaviour in fibre reinforced composites. Composites Science and Technology, 2011.

[22] W. Tan, F. Naya, L. Yang, T. Chang, B. G. Falzon, L. Zhan, J. M. Molina-Aldareguía, C. González, and J. Llorca. The role of inter- facial properties on the intralaminar and interlaminar damage behaviour of unidirectional composite laminates: Experimental characterization and multiscale modelling. Composites Part B: Engineering, 2018. ISSN 1359-8368.

[23] C. Willberg, L. Wiedemann, and M. Rädel. A mode-dependent energy-based damage model for peridynamics and its implementation. Journal of Mechanics of Materials and Structures, 14(2):193-217, 2019. ISSN 1559-3959.

[24] Dong Yang, Xiaoqiao He, Xuefeng Liu, Yajie Deng, and Xiaohua Huang. A peridynamics-based cohesive zone model (PD-CZM) for predicting cohesive crack propagation. International Journal of Mechanical Sciences, 184:105830, 2020. ISSN 0020-7403.

[25] S. Zhandarov and E. Mäder. Analysis of a pull-out test with real specimen geometry. Part II: the effect of meniscus. Journal of Adhesion Science and Technology, 2014.

[26] S. Zhandarov and E. Mäder. Analysis of a pull-out test with real specimen geometry. Part I: matrix droplet in the shape of a spherical segment. Journal of Adhesion Science and Technology 2013.

[27] S. Zhandarov and E. Mäder. An alternative method of determining the local interfacial shear strength from force-displacement curves in the pull-out and microbond tests. International Journal of Adhesion and Adhesives, pages 37-42, 2014.

[28] S. Zhandarov and E. Mäder. Determining the interfacial toughness from force-displacement curves in the pull-out and microbond tests using the alternative method. International Journal of Adhesion and Adhesives, 65:11-18, 2016.

[29] S. Zhandarov, E. Pisanova, and E. Mäder. Is there any contradiction between the stress and energy failure criteria in micromechanical tests? part ii. crack propagation: Effect of friction on force-displacement curves. Composite Interfaces, 7(3):149-175, 2012.

[30] S. Zhandarov, E. Madenci, C. Scheffler, G. Kalinka, C. Poitzsch, and S. Fliescher. Investigation of interfacial strength parameters in polymer matrix composites: Compatibility and reproducibility. Advanced Industrial and Engineering Polymer Research, pages 82-92, 2018.

[31] S. Zhandarov, E. Mäder, and U. Gohs. Why should the "alternative" method of estimating local interfacial shear strength in a pull-out test be preferred to other methods? materials, 2018.

[32] Y. Zhang and P. Qiao. Peridynamic simulation of twodimensional axisymmetric pull-out tests. International Journal of Solids and Structures, pages 41-57, 2019. 